Pacific Journal of Mathematics

ONE-SIDED HEEGAARD SPLITTINGS OF 3-MANIFOLDS 


\title{
ONE-SIDED HEEGAARD SPLITTINGS OF 3-MANIFOLDS
}

\section{J. H. RUBINSTEIN}

\begin{abstract}
For a large class of closed orientable 3-manifolds, we define a new decomposition method which uses embedded one-sided surfaces and is analogous to Heegaard splittings. The technique is most useful for studying some "small" 3manifolds (i.e., which have finite fundamental group or are not sufficiently large). We give several general criteria for existence of these splittings and some results on nonorientable surfaces in lens spaces. Also stable equivalence (as for Heegaard splittings) and a result of Waldhausen's are shown to carry over to the one-sided case.
\end{abstract}

O. Introduction. In [7] an example is given showing that the loop theorem is not valid for one-sided surfaces in 3-manifolds. For this reason, such surfaces are difficult to handle and have not been the object of much work. We would like to present a new approach based on the following:

Definition. Let $M$ be a closed orientable 3-manifold. A pair $(M, K)$ is called a one-sided Heegaard splitting if $K$ is a closed nonorientable surface embedded in $M$ such that $M-K$ is an open handlebody.

REMARKs. (1) If $(M, K)$ is a one-sided Heegaard splitting and if $N(K)$ is a small closed regular neighborhood of $K$, then $N(K)$ is homeomorphic to a twisted line-bundle over $K$. Also $M$-int $N(K)$ is a handlebody which we denote by $Y$.

(2) There is a double cover $p: \widetilde{M} \rightarrow M$ naturally associated with a one-sided Heegaard splitting $(M, K)$. It is the covering of $M$ corresponding to the subgroup $i_{*} \pi_{1}(M-K)$ of $\pi_{1}(M)$, where $i$ is the inclusion. The surface $p^{-1}(K)$, which we denote by $\widetilde{K}$, is the orientable double cover of $K$ and $(\widetilde{M}, \widetilde{K})$ is a Heegaard decomposition (i.e., the closures of the components of $\widetilde{M}-\widetilde{K}$ are handlebodies). If $g: \widetilde{M} \rightarrow \widetilde{M}$ is the covering transformation for $p$ then $\widetilde{K}$ is $g$ invariant and $g$ interchanges the components of $\widetilde{M}-\widetilde{K}$.

We work in the PL category and let $M$ be a closed orientable 3-manifold throughout. In $\S 1$ it is proved that there are one-sided splittings associated with any nonzero class in $H_{2}\left(M, Z_{2}\right)$. In $\S 2$ one-sided decompositions are discussed where the surface $K$ is (geometrically) incompressible. This is the most useful setting for the theory (cf. [4] for some results employing this approach). 
In $\S 3$ a method based on [1] is given for studying the relation in a lens space between a genus 1 Heegaard splitting and an embedded incompressible nonorientable surface. As applications, it is proved that any two incompressible surfaces in a lens space are isotopic and a simple derivation of the algorithm of [1] for the genus of these surfaces is given.

In $\S 4$ we show that two one-sided splittings associated with the same class in $H_{2}\left(M, Z_{2}\right)$ are stably equivalent (cf. [6]). Finally in $\S 5$ the standard form for maps of degree 1 in [9] is adapted to one-sided decompositions.

\section{Existence of one-sided splittings.}

Definition. Suppose $K$ is a closed nonorientable surface embedded in $M$. We denote by $[K]$ the class in $H_{2}\left(M, Z_{2}\right)$ which is the image of the generator of $H_{2}\left(K, Z_{2}\right)$ by the map induced by the embedding of $K$ in $M$. If $(M, K)$ is a one-sided Heegaard splitting then we call $[K]$ the class associated with $(M, K)$.

Theorem 1. For any element $\alpha \neq 0$ in $H_{2}\left(M, Z_{2}\right)$, there is a one-sided Heegaard splitting $(M, K)$ with $[K]=\alpha$.

Proof. The existence of a closed nonorientable surface $K$ embedded in with $[K]=\alpha$ follows as in [1]. Explicitly let $f: M \rightarrow R P^{4}$ be a map such that $f_{\sharp}: H_{1}\left(M, Z_{2}\right) \rightarrow H_{1}\left(R P^{4}, Z_{2}\right)$ gives the element of $H^{1}\left(M, Z_{2}\right)$ corresponding to $\alpha$ by Poincaré duality. Assume $f$ is transverse on the submanifold $R P^{3}$. We can then join up the components of the surface $f^{-1}\left(R P^{3}\right)$ using elementary surgery, by a homotopy of $f$. This gives $K$.

As in Remark 2 above, there is a double cover $p: \tilde{M} \rightarrow M$ associated with $K$. Suppose $M$ is triangulated with $K$ as a full subcomplex. Then there is a lifted $g$-invariant triangulation of $\tilde{M}$. Let $\widetilde{K}=p^{-1}(K)$ and let the vertices in $\widetilde{K}$ be divided into disjoint classes $V$ and $W$ with $W=g V$. We denote the closures of the components of $\tilde{M}-\widetilde{K}$ by $Y_{1}$ and $Y_{2}$.

Let $\Gamma_{i}$ be the union of the 1-simplices of the triangulation of $\widetilde{M}$ with interior in int $Y_{i}, i=1,2$. Let $N\left(\Gamma_{i}\right)$ denote the closed simplicial regular neighborhood of $\Gamma_{i}$ in $Y_{i}$, with respect to the second derived triangulation. Then $Y_{i}$ - int $N\left(\Gamma_{i}\right)$ is the simplicial neighborhood of the dual 1-skeleton of $Y_{i}$ and so is a handlebody.

Clearly $\quad N\left(\Gamma_{1}\right) \cap \widetilde{K}=N\left(\Gamma_{2}\right) \cap \widetilde{K}=N(V) \cup N(W)$, with $N(V) \cap$ $N(W)=\varnothing$ and $g N(V)=N(W)$. Let $\bar{N}(V)$ be a small closed regular neighborhood of $N(V)$ in $Y_{1}$, chosen so that $\bar{N}(V) \cap g \bar{N}(V)=\varnothing$. By an ambient isotopy of $Y_{1}$ with support in $\bar{N}(V)$, we can shift 
$\Gamma_{1}$ to $\Gamma_{1}^{*}$ and $N\left(\Gamma_{1}\right)$ to $N\left(\Gamma_{1}^{*}\right)$ so that $N\left(\Gamma_{1}^{*}\right) \cap g N\left(\Gamma_{1}^{*}\right)=\varnothing$. We denote $g \Gamma_{1}^{*}$ by $\Gamma_{2}^{*}$ and $g N\left(\Gamma_{1}^{*}\right)$ by $N\left(\Gamma_{2}^{*}\right)$.

Define $Y_{1}^{*}$ as $\left(Y_{1}-\operatorname{int} N\left(\Gamma_{1}^{*}\right)\right) \cup N\left(\Gamma_{2}^{*}\right)$. Then $Y_{1}^{*}$, which is obtained by adding the handles of $N\left(\Gamma_{2}^{*}\right)$ to the handlebody $Y_{1}$ int $N\left(\Gamma_{1}^{*}\right)$, is a handlebody. Also $\widetilde{K}^{*}=\partial Y_{1}^{*}$ is $g$-invariant and so $\left(\widetilde{M}, \widetilde{K}^{*}\right)$ is a $g$-invariant Heegaard splitting. Therefore $\left(M, K^{*}\right)$ is a one-sided Heegaard splitting, where $K^{*}=p\left(\widetilde{K}^{*}\right)$. It is clear that $[K]=\left[K^{*}\right]$, since the same double covering $\widetilde{M}$ is associated with $K$ and $K^{*}$.

Note. $\quad H_{2}\left(M, Z_{2}\right) \cong H^{1}\left(M, Z_{2}\right) \cong H_{1}\left(M, Z_{2}\right)$. So $M$ has a one-sided Heegaard splitting if and only if $H_{1}\left(M, Z_{2}\right) \neq 0$.

\section{Incompressible one-sided splittings.}

Definition. A closed surface $K \neq S^{2}$ embedded in $M$ is incompressible if there is no disk $D$ embedded in $M$ with $D \cap K=\partial D$ a noncontractible curve in $K$.

REMARK. If $K \neq S^{2}$ is two-sided in $M$, then as is well-known by the loop theorem, $K$ is incompressible if and only if the map $i_{*}: \pi_{1}(K) \rightarrow \pi_{1}(M)$ induced by the embedding is an injection. No similar such a nice criterion has been found for one-sided surfaces.

Definition. $M$ is irreducible if any 2-sphere embedded in $M$ bounds a 3-cell.

LemMa 2. Suppose $M$ is irreducible and $K$ is an incompressible nonorientable surface embedded in $M$. The following are equivalent:

(1) $M-K$ is an open handlebody.

(2) The map $i_{*}: \pi_{1}(K) \rightarrow \pi_{1}(M)$ is onto.

(3) There is no incompressible orientable surface in $M$ which is disjoint from $K$.

Proof. $1 \Rightarrow 2$ is clear.

$2 \Rightarrow 3$. Suppose $J$ is an orientable incompressible surface embedded in $M-K$ and $i_{*}$ is onto. If $J$ is nonseparating in $M$ then there is a loop $C$ which intersects $J$ in a single point. But $C$ is homotopic to a loop in $K$, because $\pi_{1}(K) \rightarrow \pi_{1}(M)$ is onto. Therefore since $J \cap K=\varnothing$, we get a contradiction to the invariance of intersection number under homotopy.

On the other hand if $J$ is separating in $M$ then $J=\partial W$ with $W$ contained in $M-K$. As $i_{*}$ is onto, it follows that $\pi_{1}(J) \rightarrow \pi_{1}(W)$ is onto. This implies that $J$ is a 2 -sphere (since as is well-known, 
$\left.2 \beta_{1}(W) \leqq \beta_{1}(\partial W)\right)$, contrary to our assumption.

$3 \Longrightarrow 1$. Let $N(K)$ be a small closed regular neighborhood of $K$ with $Y=M-\operatorname{int} N(K)$ and $L=\partial N(K)$. We compress $L$ to get $L_{1}$, each of whose components is incompressible or is a 2-sphere. All the compressions can be made to occur in $Y$ since $K$ is incompressible, as in 14.12 of [3]. Consequently we obtain that $L_{1}$ is contained in $Y$.

By assumption there are no incompressible orientable surfaces in $Y$. Therefore we conclude that $L_{1}$ is a union of 2 -spheres. Then as $M$ is irreducible, these 2-spheres bound 3-cells and it easily follows that $Y$ is a handlebody.

Lemma 3. Suppose $H_{1}(M, Z)$ is finite. If $\alpha \neq 0$ is in $H_{2}\left(M, Z_{2}\right)$ then there are disjoint nonorientable incompressible surfaces $K_{i}$ in $M$ with $\sum_{i=1}^{n}\left[K_{i}\right]=\alpha$. In particular there is a generating set of classes of $H_{2}\left(M, Z_{2}\right)$ represented by such surfaces.

Proof. As in Theorem 1, given $\alpha$ there is a closed nonorientable surface $K$ embedded in $M$ with $[K]=\alpha$. We compress $K$ to $K_{1}$ satisfying each component is incompressible or a 2-sphere. If some two-sided component of $K_{1}$ is nonseparating in $M$, then a loop meeting this surface in a single point gives an element of infinite order in $H_{1}(M, Z)$, contrary to assumption. So all such components are null-homologous and the one-sided components have associated classes in $H_{2}\left(M, Z_{2}\right)$ which sum to give $\alpha$. We conclude that among all such classes there is a set of generators for $H_{2}\left(M, Z_{2}\right)$.

EXAMPLE. $S^{2} \times S^{1}$ has no embedded incompressible nonorientable surfaces, though by Theorem 1 it has one-sided Heegaard splittings.

Proof. Suppose $K$ is incompressible and one-sided in $S^{2} \times S^{1}$. Assume $K$ is transverse to $S^{2} \times\{x\}$. Then by induction on the number of curves of $K \cap S^{2} \times\{x\}$, it is easy to achieve $S^{2} \times\{x\} \cap$ $K=\varnothing$ by an isotopy of $K$. So $K$ is contained in a 3-cell which is impossible.

DeFINITION. We call $(M, K)$ an incompressible one-sided Heegaard splitting if $(M, K)$ is a one-sided Heegaard splitting and $K$ is incompressible in $M$.

THeOREM 4. Suppose $M$ is irreducible and $H_{1}(M, Z)$ is finite. The following are equivalent:

(1) For any orientable incompressible surface $J$ embedded in $M$, the $\operatorname{map} H_{1}\left(J, Z_{2}\right) \rightarrow H_{1}\left(M, Z_{2}\right)$ induced by the inclusion is onto. 
(2) For any nonorientable incompressible surface $K$ embedded in $M, M-K$ is an open handlebody.

If these conditions are satisfied, then there is an incompressible one-sided splitting associated with any nonzero class in $H_{2}\left(M, Z_{2}\right)$.

Proof. $1 \Rightarrow 2$. Suppose $K$ is nonorientable and incompressible in $M$. If $J$ is orientable, incompressible and disjoint from $K$ in $M$, then the image of $H_{1}\left(J, Z_{2}\right) \rightarrow H_{1}\left(M, Z_{2}\right)$ is in the kernel of the epimorphism $H_{1}\left(M, Z_{2}\right) \rightarrow Z_{2}$ which is the element of $H^{1}\left(M, Z_{2}\right)$ dual to [K]. So the map $H_{1}\left(J, Z_{2}\right) \rightarrow H_{1}\left(M, Z_{2}\right)$ is not onto, contrary to assumption. We conclude that $J \cap K=\varnothing$ does not occur and therefore by Lemma $2, M-K$ is an open handlebody.

$2 \Rightarrow 1$. Suppose $J$ is an orientable and incompressible surface in $M$, for which $H_{1}\left(J, Z_{2}\right) \rightarrow H_{1}\left(M, Z_{2}\right)$ is not onto. Then there is an epimorphism $H_{1}\left(M, Z_{2}\right) \rightarrow Z_{2}$ with kernel containing the image of $H_{1}\left(J, Z_{2}\right)$. Let $f: M \rightarrow R P^{4}$ be a map realizing the epimorphism. Since $f$ restricted to $J$ is null-homotopic, without loss of generality we can assume $f$ is transverse on $R P^{3}$ and $f(J) \cap R P^{3}=\varnothing$.

Because $H_{1}(M, Z)$ is finite, all the two-sided components of $f^{-1}\left(R P^{3}\right)$ are separating and there must be some one-sided components. As in Lemma 3, we compress one of these latter surfaces to obtain a one-sided incompressible surface $K$. Since $J$ is incompressible and $M$ is irreducible, all the compressions can be made to occur in $M-J$. By assumption $M-K$ is an open handlebody containing $J$ and this gives a contradiction.

We now consider the last assertion of the theorem. Since $H_{1}(M, Z)$ is finite, given $\alpha \neq 0$ in $H_{2}\left(M, Z_{2}\right)$ Lemma 3 shows there are disjoint nonorientable incompressible surfaces $K_{i}$ in $M$ with $\sum_{i=1}^{n}\left[K_{i}\right]=\alpha$. But for each of these, $M-K_{i}$ is an open handlebody by Condition 2. For a handlebody $X$, since $H_{2}\left(X, Z_{2}\right)=0$ it follows that there are no closed nonorientable surfaces embedded in $X$. We conclude that $n=1$ and $\left[K_{1}\right]=\alpha$ as desired.

Corollary 5. Suppose $M$ is irreducible and not sufficiently large. Then there is an incompressible one-sided splitting associated with any nonzero class in $H_{2}\left(M, Z_{2}\right)$.

REMARK. The theorem shows that $M$ has many incompressible one-sided splittings if and only if the existence of incompressible two-sided surfaces is restricted.

Proposition 6. Suppose $M$ has an incompressible one-sided Heegaard splitting. Then $M$ is irreducible.

Proof. Suppose $S$ is a 2-sphere embedded in $M$. Assume $S$ is 
transverse to $K$, where $(M, K)$ is an incompressible one-sided splitting. Since $M-K$ is irreducible, it follows easily by induction on the number of curves of $S \cap K$ that $S$ bounds a 3-cell.

THEOREM 7. Suppose $M$ is an orientable Seifert manifold with fiber giving a nonzero class in $H_{1}\left(M, Z_{2}\right)$. Assume $M$ is neither a union of two twisted line-bundles over a nonorientable surface nor a surface-bundle over a circle. Then $M$ has an incompressible onesided Heegaard splitting.

Proof. For the properties of Seifert manifolds, see [5]. By Proposition 1 of [2], $M$ is irreducible. Let $h$ be a fiber of $M$. Since $[h] \neq 0$ in $H_{1}\left(M, Z_{2}\right)$, there is an epimorphism $H_{1}\left(M, Z_{2}\right) \rightarrow Z_{2}$ with [h] not in the kernel. As in Theorem 1, we can find a closed nonorientable surface $K$ embedded in $M$ with $[K]$ dual to the corresponding element of $H^{1}\left(M, Z_{2}\right)$. Consequently $K$ and $h$ have odd intersection number.

Compressing $K$, we get a component $K_{1}$ which has odd intersection number with $h$ and is either a 2-sphere or is incompressible in $M$. Suppose that the orbit surface for $M$ is nonorientable. Then we lift $K_{1}, h$ to $\widetilde{K}_{1}, \widetilde{h}$ in a double covering $\tilde{M}$ of $M$, so that $\tilde{M}$ has an orientable orbit surface. Since $\widetilde{h}$ covers $h$ once, it follows that $\widetilde{K}_{1}, \widetilde{h}$ also have odd intersection number. The homotopy class $\{\widetilde{h}\}$ is central in $\pi_{1}(\widetilde{M})$. There are two possibilities.

Case 1. $\widetilde{K}_{1}$ is two-sided in $\tilde{M}$.

After compressing $\widetilde{K}_{1}$ if necessary, we obtain a two-sided incompressible surface $J$ which has odd intersection number with $\tilde{h}$. Therefore $\{\tilde{h}\}$ is not in the image of $\pi_{1}(J)$ in $\pi_{1}(\tilde{M})$. Exactly as in Theorem 2 of [2] it follows that $\tilde{M}$ is a $J$-bundle over $S^{1}$ and $M$ is either a union of two twisted line-bundles over a nonorientable surface, or a surface-bundle over $S^{1}$, contrary to assumption.

Case 2. $\widetilde{K}_{1}$ is one-sided in $\tilde{M}$.

Then $K_{1}$ is one-sided in $M$. Suppose that $J$ is an orientable incompressible surface in $M-K_{1}$. Since $h$ and $K_{1}$ have an odd intersection number, we see that $\{h\}$ is not in the image of $\pi_{1}(J)$ in $\pi_{1}(M)$. Passing to $\tilde{M}$, we can apply the same argument as in Case 1. This gives a contradiction and so by Lemma 2, we conclude that $M-K_{1}$ is a handlebody.

If the orbit surface for $M$ is orientable then a similar method applies.

REMARKs. (1) Exactly the same argument as in (4.6) of [1] 
can be employed to show that if $M$ is an orientable Seifert manifold with orientable orbit surface and if $K$ is an embedded nonorientable surface which has odd intersection number with the fiber in $M$, then $\pi_{1}(K) \rightarrow \pi_{1}(M)$ is onto.

(2) To get examples where $[h] \neq 0$ in $H_{1}\left(M, Z_{2}\right)$, take $M$ such that all the $\mu$-invariants (multiplicities of the exceptional fibers) are odd and the $b$-invariant differs from the sum of the $\nu$-invariants by an even number.

(3) The 3-manifolds in this theorem are in general sufficiently large. They contain incompressible tori formed by the ordinary fibers projecting to suitable circles in the orbit surface. Note that such surfaces to some extent satisfy the conditions of Theorem 4; the image of $H_{1}\left(T, Z_{2}\right)$ in $H_{1}\left(M, Z_{2}\right)$ certainly contains [h], where $T$ is a torus of ordinary fibers.

Proposition 8. Let $M$ be a Seifert manifold with $S^{2}$ as orbit surface and exceptional fibers of multiplicity $(2,2, n)$ excluding $S^{2} \times S^{1}$, or $(2,4, n)$, for $n \geqq 1$. Then there is an incompressible one-sided splitting $(M, K)$ such that $K$ has genus 2 or 3 respectively.

Proof. For the $(2,2, n)$ case, see [4]. In the other case let $N_{1}$ and $N_{2}$ be small fibered neighborhoods of the exceptional fibers of multiplicity 2 and 4 respectively and let $\Phi$ be the projection from $M$ to the orbit surface. Choose a nonsingular arc $\lambda$ in the orbit surface from $\Phi\left(\partial N_{1}\right)$ to $\Phi\left(\partial N_{2}\right)$ missing the image of the third exceptional fiber and $\Phi$ (int $N_{1} \cup$ int $\left.N_{2}\right)$. Then $\Phi^{-1}(\lambda)$ is an annulus in $M$ with $\partial \Phi^{-1}(\lambda)=\Phi^{-1}(\partial \lambda)$ consisting of a fiber in $\partial N_{1}$ and one in $\partial N_{2}$. By [1] or $\S 3$ it follows that the component of $\partial \Phi^{-1}(\lambda)$ in $\partial N_{1}$ bounds a Mobius band in $N_{1}$, while the other curve of $\partial \Phi^{-1}(\lambda)$ bounds a Klein bottle with a hole in $N_{2}$. Therefore we obtain a nonorientable surface $K$ of genus 3 embedded in $M$.

If $K$ is compressible in $M$ then $M$ contains an embedded onesided $R P^{2}$. By Proposition 1 of [2], $M \approx R P^{3}$ or $M \approx R P^{3} \sharp R P^{3}$ follows. The presentation of $\pi_{1}(M)$ given by the fibering (see [5]) shows that $\pi_{1}(M)=Z_{2}$ or $Z_{2} * Z_{2}$ is impossible. So $K$ is incompressible.

Finally we prove that $M-K$ is an open handlebody. Now it is easy to see as in $\S 3$ or [1] that the map $\pi_{1}(K) \rightarrow \pi_{1}\left(N_{1} \cup N_{2} \cup \Phi^{-1}(\lambda)\right)$ induced by the inclusion is onto. Since the complement of $N_{1} \cup N_{2} \cup$ $\Phi^{-1}(\lambda)$ in $M$ is an open solid torus, it follows that $\pi_{1}(K) \rightarrow \pi_{1}(M)$ is onto and so by Lemma 2 the result is established.

REMARKs. (1) Note that a Seifert space with exceptional fibers of multiplicity $(2,2,4)$ and $S^{2}$ as orbit surface has incompressible 
splittings of genus 2 and genus 3 . These are associated with different classes in $H_{2}\left(M, Z_{2}\right)$ however.

(2) The Seifert manifolds with $S^{2}$ as orbit surface and exceptional fibers of multiplicity $(2,2, n)$ excluding $S^{1} \times S^{2}$, or $(2,4,3)$, all have finite fundamental groups.

3. One-sided surfaces in lens spaces. Let $M$ be the lens space $L(2 k, q)$, where $(2 k, q)=1$. Since $H_{2}\left(M, Z_{2}\right)=Z_{2}, M$ contains embedded nonorientable surfaces. In [1] an algorithm is given for the minimal genus, denoted $N(2 k, q)$, of such surfaces. Note that an embedded nonorientable surface of minimal genus is incompressible, since $H_{2}(M, Z)=0$.

Let $K$ denote an incompressible surface in $M$ throughout this section. Since $M$ is not sufficiently large, $K$ must be nonorientable and $M-K$ is an open handlebody by Corollary 5 . Let $(M, L)$ be a Heegaard splitting of genus 1 , with $M=T \cup T^{\prime}$ where $T \cup T^{\prime}=$ $\partial T=\partial T^{\prime}=L$ and $T, T^{\prime}$ are solid tori.

Lemma 9. After an isotopy, $K \cap T$ can be transformed into a disk.

Proof. (cf. [1, p. 99]). By shrinking $T$, it can be assumed that $K \cap T$ is a collection of $n$ meridian disks for $T$. Let $D^{\prime}$ be a meridian disk for $T^{\prime}$. By an isotopy of $K$, we can suppose that $D^{\prime}$ is transverse to $K$ and $K \cap D^{\prime}$ contains no simple closed curves, since $K$ is incompressible. Also without loss of generality $D^{\prime}$ can be chosen so that no arc in $\partial D^{\prime}$ together with an arc in $K \cap L$ bounds a disk in $L$.

Let $\lambda$ be a component of $K \cap D^{\prime}$. Then $\lambda$ is an arc with ends on curves of $K \cap L$. If $\mu$ is an arc of $\partial D^{\prime}$ with $\partial \lambda=\partial \mu$ then we choose $\lambda$ so that (int $\mu) \cap K=\varnothing$.

Case 1. Both ends of $\lambda$ are on a curve $C$ of $K \cap L$.

Then either $n=1$ or there is an arc $\gamma$ of $C$ with $\partial \mu=\partial \gamma$ and $\mu \cup \gamma$ bounds a disk on $L$. The latter contradicts our choice of $D^{\prime}$.

Case 2. $\lambda$ joins curves $C$ and $C^{\prime}$ of $K \cap L$.

There is an isotopy of $K$ with support in a neighborhood of $D^{\prime}$, which carries $\lambda$ to $\mu$ along $D^{\prime}$. After this isotopy $K$ meets $T$ in $n-2$ meridian disks and in a disk parallel to a disk on $L$. So a further isotopy gives $n-2$ meridian disks in $K \cap T$ and the result follows by induction on $n$. Note $K \cap T=\varnothing$ implies $K$ is contained in $T^{\prime}$, which is impossible.

Lemma 10. By an isotopy, $K \cap T$ can be changed into a Mobius 
band.

Proof. By Lemma 9 we can assume $K \cap T$ is a single meridian disk. Denote $K \cap L$ by $C$ and let $D^{\prime}$ be a meridian disk for $T^{\prime}$. We can again suppose that $K$ is transverse to $D^{\prime}$, there are no closed curves in $K \cap D^{\prime}$ and no arc in $\partial D^{\prime}$ together with an arc in $C$ bounds a disk in $L$.

Let $\lambda$ be an arc of $K \cap D^{\prime}$, chosen so that an arc $\mu$ of $\partial D^{\prime}$ with $\partial \mu=\partial \lambda$ satisfies (int $\mu$ ) $\cap K=\varnothing$. If $\mu$ has both ends on the same side of $C$, then we get a contradiction to our choice of $D^{\prime}$. Hence $\mu$ goes from one side of $C$ to the other. The isotopy of $K$ taking $\lambda$ to $\mu$ along $D^{\prime}$ transforms $K \cap T$ into a Mobius band as required.

Proposition 11. There are disjoint tori $L_{j}, 1 \leqq j \leqq g-1$, in $M(g \doteq$ genus $K)$ which are all parallel to each other and give Heegaard splittings. The tori satisfy $L_{j} \cap K=C_{j}$ is a single curve and $K-\cup_{j} C_{j}$ consists of 2 open Mobius bands and $g-2$ open Mobius bands with single holes.

Proof. By Lemma 10 there is a torus $L_{1}$ with $L_{1} \cap K=C_{1}$ and $T_{1} \cap K$ equal to a Mobius band, where $L_{1}=\partial T_{1}=\partial T_{1}^{\prime}$ and $T_{1}, T_{1}^{\prime}$ are solid tori. We can assume $K$ is transverse to $D_{1}^{\prime}$, a meridian disk for $T_{1}^{\prime}, K \cap D_{1}^{\prime}$ contains no loops and no arc in $\partial D_{1}^{\prime}$ together with an arc in $C_{1}$ bounds a disk in $L_{1}$. Then arcs $\mu$ and $\lambda$ can be found with properties as in Lemma 10. Let $L_{2}$ be the image of $L_{1}$ by the isotopy taking $\mu$ to $\lambda$ along $D_{1}^{\prime}$ and pushing $L_{1}$ a small distance into int $T_{1}^{\prime}$. Then $L_{2}$ has the required properties and all the tori $L_{j}$ are constructed similarly. Note that after $g$ steps the meridian disk $D_{g}^{\prime}$ can be chosen to miss $K$ and the process stops.

THEOREM 12. Any two incompressible surfaces embedded in $M$ are isotopic.

Proof. Suppose $K_{1}, K_{2}$ are both incompressible surfaces in $M$ and let $(M, L)$ be a Heegaard splitting of genus 1 with $L=\partial T=\partial T^{\prime}$. By Lemma 9 we can assume $K_{1} \cap T$ and $K_{2} \cap T$ are disjoint meridian disks. Let $C_{i}$ denote the curve $K_{i} \cap L, i=1,2$.

Let $D^{\prime}$ be a meridian disk for $T^{\prime}$ and apply the argument for Lemma 10. Then $\operatorname{arcs} \mu_{i}, \lambda_{i}$ with the properties in that proof for $K_{i}$ can be found, $i=1,2$. Without loss of generality we can suppose that no arc of $\partial D^{\prime}$ with interior disjoint from $C_{i}$ has both ends on the same side of $C_{i}$, for $i=1$ or 2 . It can be assumed also that $\mu_{1} \cap C_{2}$ and $\mu_{2} \cap C_{1}$ are both single points. There are three possibilities as shown in Figure 1. 


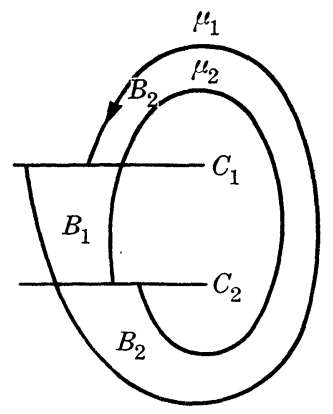

(A)

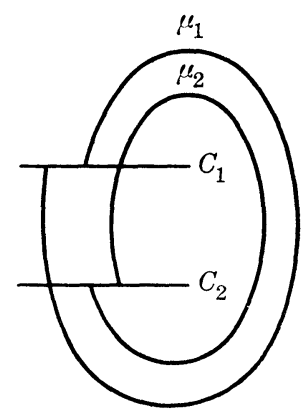

(B)

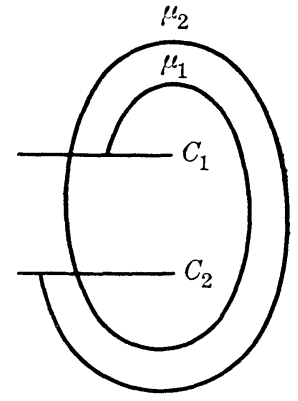

(C)

FIGURE 1

In (A) $\mu_{1}$ and $\mu_{2}$ "spiral" oppositely, while in (B) and (C) they "spiral" in the same direction. Note that in (C), $\mu_{1} \cap \mu_{2} \neq \varnothing$. It is easy to see that in cases (B) and (C), after the isotopies of $K_{t}$ taking $\lambda_{i}$ to $\mu_{i}$ along $D^{\prime}$, the new curves $K_{i} \cap L$ are isotopic on $L$. Consequently by a further isotopy it can be achieved that $K_{1} \cap T$ and $K_{2} \cap T$ are equal to the same Mobius band.

Suppose on the other hand that (A) is true. The four components of $L-C_{1}-C_{2}-\mu_{1}-\mu_{2}$ are open disks. Let $B_{1}$ and $B$, be the closures of two of these, chosen as shown in (A) of Figure 1. Assume that $\partial D^{\prime}$ is oriented so that it enters $B_{1}$ at the end-point of $\mu_{1}$. Then $\partial D^{\prime}$ must leave $B_{1}$ at a point of $C_{2} \cap B_{2}$, since otherwise there is an arc of $\partial D^{\prime}$ with both ends on the same side of $C_{1}$, contrary to assumption. So $\partial D^{\prime}$ enters $B_{2}$ and similarly leaves it at a point of $C_{1} \cap B_{1}$. This implies that $\partial D^{\prime}$ remains in $B_{1} \cup B_{2}$, which is clearly false. We conclude that (A) does not occur.

To continue the procedure, it is only necessary to slightly disjoin $K_{1}$ and $K_{2}$ near $L$ by an isotopy. After $g$ steps it follows that $K_{1}$ and $K_{2}$ are isotopic.

Definition. One-sided Heegaard splittings $\left(M, K_{1}\right)$ and $\left(M, K_{2}\right)$ are strongly equivalent if $K_{1}$ and $K_{2}$ are isotopic.

REMARKs. (1) The theorem implies that any incompressible surface in a lens space has minimal genus.

(2) An equivalent statement to the theorem is that any two incompressible one-sided splittings of a lens space are strongly equivalent. We conjecture that this is true for arbitrary one-sided splittings for which the surfaces have the same genus in a lens space [cf. [10]).

(3) Let $M$ be a lens space and $(M, K)$ be an incompressible one-sided splitting. Let $\mathscr{H}(M)$ denote the quotient of the homeomorphism group of $M$ by the normal subgroup of homeomorphisms isotopic to the identity. The theorem implies that any class in 
$\mathscr{X}(M)$ is represented by a homeomorphism which preserves the splitting $(M, K)$. This can be useful for studying $\mathscr{C}(M)$ (cf. [4] for the case $M=L(4 n, \pm(2 n-1)))$.

TheOREM 13 ((8.3) of [1]). $\quad N(2 k, q)=1+N(2(k-Q), q-2 m)$, where $m, Q$ satisfy $2 k m-q Q= \pm 1,0<Q<k$.

Proof. Let $K$ be an incompressible surface in $M$. By Lemma 10 we can assume $K \cap T$ is a Mobius band denoted $E$, where as usual $M=T \cup T^{\prime}, T \cap T^{\prime}=L$ and $T, T^{\prime}$ are solid tori. Let $T_{1}$ be a solid torus with meridian disk $D_{1}$. We form a new lens space $M_{1}$ as the union of $T_{1}$ and $T^{\prime}$ identified along their boundaries by a homeomorphism which takes $\partial D_{1}$ to $\partial E$. Then $M_{1}$ contains a nonorientable surface $K_{1}=(K-\operatorname{int} E) \cup D_{1}$, which has genus 1 less than $K$.

Suppose $K_{1}$ is compressible in $M_{1}$. Then there is a nonorientable surface $K_{2}$ embedded in $M_{1}$ with smaller genus than $K_{1}$. By Lemma 9, after an isotopy $K_{2} \cap T_{1}$ can be transformed into a meridian disk. But then $K_{2}$ - int $T_{1}$ has a boundary curve which bounds a Mobius band in $T$. Consequently there is a nonorientable surface in $M$ with genus 1 greater than $K_{2}$, which contradicts our assumption that $K$ has minimal genus. Therefore if $M_{1}=L\left(2 k_{1}, q_{1}\right)$ we conclude that $N(2 k, q)=1+N\left(2 k_{1}, q_{1}\right)$.

In $H_{1}(L, Z)$ let $a, a^{\prime}$ be elements satisfying $a \sim 0$ in $T$ and $a^{\prime} \sim 0$ in $T^{\prime}$. Also let classes $b, b^{\prime}$ be chosen so that $\{a, b\},\left\{a^{\prime}, b^{\prime}\right\}$ are bases for $H_{1}(L, Z)$. Without loss of generality we can suppose that $a=$ $q a^{\prime}+2 k b^{\prime}$ and $b=m a^{\prime}+Q b^{\prime}$, with $m, Q$ chosen as above, since $M=L(2 k, q)$.

Now $a, a^{\prime}$ have intersection number $\pm 2 k$. In the argument for Lemma 10, clearly $\partial E$ has two less intersection points with the curve $\partial D^{\prime}$ than $\partial D$ does, where $D, D^{\prime}$ are the meridian disks for $T, T^{\prime}$ respectively. So $[\partial E]$ and $a^{\prime}$ have intersection number with absolute value strictly less than $2 k$. Also by a suitable orientation of $\partial E$ it can be assumed that $[\partial E]=-2 b+N a$ where $N$ is odd, since $\partial E$ and $\partial D$ have intersection number \pm 2 . Hence $[\partial E]=(N q-2 m) a^{\prime}+$ $(2 k N-2 Q) b^{\prime}$ and $[\partial E], a^{\prime}$ have intersection number $\pm(2 k N-2 Q)$. We conclude that $N=1$. Since $\partial E$ is a meridian curve for $T_{1}$, it follows that $M_{1}=L(2 k-2 Q, q-2 m)$ as desired.

4. Stable equivalence. Let $(M, K)$ be a one-sided Heegaard splitting and $\left(M^{\prime}, K^{\prime}\right)$ a one- or two-sided Heegaard splitting. Let $B, B^{\prime}$ be 3-cells in $M, M^{\prime}$ such that $B \cap K=D$ and $B^{\prime} \cap K^{\prime}=D^{\prime}$ are disks. Suppose $M-\operatorname{int} B$ is attached to $M^{\prime}$ - int $B^{\prime}$ by a homeomorphism of the boundary 2-spheres, which takes $\partial D$ to $\partial D^{\prime}$. The 
resulting 3-manifold is the connected sum $M \# M^{\prime}$ and contains the closed nonorientable surface $K \# K^{\prime}$. Clearly $\left(M \# M^{\prime}, K \# K^{\prime}\right)$ is a one-sided Heegaard splitting which we will denote by $(M, K) \#\left(M^{\prime}, K^{\prime}\right)$ and call the connected sum of the splittings $(M, K),\left(M^{\prime}, K^{\prime}\right)$.

Definition. One-sided Heegaard splittings $(M, K),\left(M^{\prime}, K^{\prime}\right)$ are equivalent if there is a homeomorphism from $M$ to $M^{\prime}$, which maps $K$ to $K^{\prime}$.

Note that $(M, K) \#\left(M^{\prime}, K^{\prime}\right)$ is independent of the choice of the 3-cells $B, B^{\prime}$ up to equivalence. Let $\left(S^{3}, L\right)$ denote the standard Heegaard splitting of genus 1 of $S^{3}$ and let $(M, K) \# n\left(S^{3}, L\right)$ be the connected sum with $n$ copies of $\left(S^{3}, L\right)$.

Definition. One-sided Heegaard splittings $(M, K),\left(M^{\prime}, K^{\prime}\right)$ are stably equivalent if $(M, K) \# n\left(S^{3}, L\right)$ is equivalent to $\left(M^{\prime}, K^{\prime}\right) \# m\left(S^{3}, L\right)$ for some $m, n$.

THEOREM 14. Suppose $(M, K),\left(M, K^{\prime}\right)$ are one-sided Heegaard splittings with $[K]=\left[K^{\prime}\right]$. Then they are stably equivalent.

Proof. There is a double cover $\widetilde{M}$ of $M$, associated with both splittings, as in $\S 0$. Let $\widetilde{K}, \widetilde{K}^{\prime}$ denote the lifts of $K, K^{\prime}$ to $\widetilde{M}$. Since $M-K^{\prime}$ is an open handlebody, there is a wedge of circles, denoted $\Lambda^{\prime}$, which is embedded in $M-K^{\prime}$ as a deformation retract. Assume that $\Lambda^{\prime}$ is transverse to $K$ and let $\Lambda_{1}^{\prime}, \Lambda_{2}^{\prime}$ be the lifts of $\Lambda^{\prime}$ to $\widetilde{M}$.

We do the procedure of Theorem 1 , using a triangulation of $M$ which contains $\Lambda^{\prime}$ as a subcomplex. Let $\Gamma_{i}, N\left(\Gamma_{i}\right), Y_{i}, V, W, \bar{N}(V)$ be defined as in Theorem 1, with respect to $K$. It can be supposed that the partition $V \cup W$ of the vertices of $\widetilde{K}$ is chosen so that $V$ contains $\Lambda_{2}^{\prime} \cap \widetilde{K}$. Instead of moving $\Gamma_{1}$ just in $\bar{N}(V)$, we need to use an isotopy with support in $\bar{N}(V) \cup \bar{N}\left(\Lambda_{2}^{\prime} \cap Y_{1}\right)$, where $\bar{N}\left(\Lambda_{2}^{\prime} \cap Y_{1}\right)$ is a small regular neighborhood of the second derived simplicial neighborhood of $\Lambda_{2}^{\prime} \cap Y_{1}$ in $Y_{1}$. The isotopy is chosen to shift $\Gamma_{1}$ to $\Gamma_{1}^{*}$ so that $N\left(\Gamma_{1}^{*}\right) \cap g N\left(\Gamma_{1}^{*}\right)=\varnothing$ and $N\left(\Gamma_{1}^{*}\right) \cap \Lambda_{2}^{\prime}=\varnothing$.

Defining $\tilde{K}^{*}$ as in Theorem 1 , we see that $\widetilde{K}^{*} \cap\left(\Lambda_{1}^{\prime} \cup \Lambda_{2}^{\prime}\right)=\varnothing$ has been achieved. Also by [6] it follows that $\left(M, K^{*}\right)$ and $(M, K)$ are stably equivalent, where $K^{*}=p\left(\widetilde{K}^{*}\right)$. So there is no loss of generality in replacing $(M, K)$ by $\left(M, K^{*}\right)$, which we now do. Then $K \cap \Lambda^{\prime}=\varnothing$ and hence $K$ is contained in a closed regular neighborhood $N\left(K^{\prime}\right)$ of $K^{\prime}$.

LEMMA 15. Suppose $K$ is closed nonorientable surface embedded in $N$ which is a twisted line-bundle over a closed nonorientable surface $K^{\prime}$. Then genus $K \geqq$ genus $K^{\prime}$. Equality holds if and only if $K$ is incompressible in $N$. In this case $K$ and $K^{\prime}$ are isotopic. 
Proof. We compress $K$ so that every component is either incompressible or a 2 -sphere. Since every two-sided surface in $N$ separates, some component $K_{1}$ is nonorientable. It suffices to prove that $K_{1}$ is isotopic to $K^{\prime}$.

Suppose without loss of generality that $K_{1}$ is transverse to $K^{\prime}$ and that $K_{1} \cap K^{\prime}$ contains only loops which are noncontractible in both $K_{1}$ and $K^{\prime}$ (as $K_{1}, K^{\prime}$ are incompressible). Let $N\left(K^{\prime}\right)$ be a small closed regular neighborhood with $K_{1} \cap N\left(K^{\prime}\right)$ consisting of Mobius bands and annuli. Since $H_{2}\left(N, Z_{2}\right)=Z_{2}$, it is easy to see that $K^{\prime}-K_{1}$ and $K_{1}-K^{\prime}$ are orientable.

$N$ - int $N\left(K^{\prime}\right)$ is homeomorphic to $\partial N \times I$ and will be denoted by $X$. Every component $R_{1}$ of $K_{1} \cap X$ is orientable and incompressible in $X$. By [8] it follows that there is a region $R^{\prime}$ in $\partial N\left(K^{\prime}\right)$ with $\partial R_{1}=\partial R^{\prime}$ and $R_{1}$ parallel to $R^{\prime}$. Clearly then $R_{1}$ can be chosen so that (int $\left.R^{\prime}\right) \cap K_{1}=\varnothing$. There are unique components $\bar{R}_{1}, \bar{R}^{\prime}$, of $K_{1}-K^{\prime}$ and $K^{\prime}-K_{1}$ containing $R_{1}, R^{\prime}$ respectively, and there is an isotopy of $K_{1}$ taking $\bar{R}_{1}$ to $\bar{R}^{\prime}$.

If $K_{1} \cap K^{\prime}$ consists entirely of orientation-reversing curves then the closure of $\bar{R}_{1}$ or $\bar{R}^{\prime}$ is $K_{1}$ or $K^{\prime}$. So $K_{1}$ and $K^{\prime}$ are isotopic. On the other hand if $K_{1} \cap K^{\prime}$ contains some orientation-preserving curves then there must be at least one in $\partial \bar{R}_{1}=\partial \bar{R}^{\prime}$. In this case, after the isotopy moving $\bar{R}_{1}$ to $\bar{R}^{\prime}$ we can slightly disjoint $K_{1}$ and $K^{\prime}$ so that the surfaces are again transverse and have fewer curves of intersection. By induction on the number of these curves, the lemma is proved.

Returning to the proof of the theorem, we have $K$ included in $N\left(K^{\prime}\right)$. So by the lemma there is a unique one-sided component $K_{1}$ obtained from compressing $K$ in $N\left(K^{\prime}\right)$, and $K_{1}$ is isotopic to $K^{\prime}$. Any two-sided incompressible surface in $N\left(K^{\prime}\right)$ must be parallel to $\partial N\left(K^{\prime}\right)$ by [8] and so is completely compressible in $M$. We conclude that $K^{\prime}$ can be assumed to be the result of a sequence of compressions of $K$.

Consequently without loss of generality there is a collection of disks $D_{1}, \cdots, D_{r}$ embedded in $M$ with the following properties

- The curves $\partial D_{i}$ are all contained in $K$ and form a nonseparating set of loops in $K$ (i.e., $K-\cup \partial D_{i}$ is connected).

$-D_{i} \cap D_{j}$ is a union of disks $D_{k}$ in the collection, for all $i, j$.

$-D_{i}$ intersects $K$ transversely for all $i$, and each component of $K \cap D_{i}$ is one of the curves $\partial D_{k}$.

- $K^{\prime}$ is obtained by successively compressing $K$ using the disks $D_{i}$ in order of size, starting with the smallest.

Next the complexity $n_{i}$ of a disk $D_{i}$ is defined to be the number of disks $D_{j}$ which are contained in $D_{i}$. Our aim is to decrease all the complexities to zero. Suppose there is a complexity which is 
nonzero. $N(K)$ can be chosen small enough so that every component of $D_{i} \cap N(K)$ is a small annulus containing exactly one curve of $D_{i} \cap K$, for all $i$.

At least one of the components of $D_{i} \cap Y$ is not a disk, for some $i$. Let us consider the intersections of the meridian disks for $Y$ with the disks $D_{i}$. Note that in a handlebody, a loop which is disjoint from a complete set of meridian disks must be contractible. Therefore it easily follows that for some $i$, either one of the components of $D_{i} \cap Y$ is compressible in $Y$ or there is a disk $D$ embedded in $Y$ with $\partial D=\lambda \cup \gamma$, where $\lambda, \gamma$ are arcs in $\partial Y, D_{i}$ respectively and both join two different curves of $D_{i} \cap \partial Y$. In the former case it is easy to see that we can alter the choice of the compressing disks $D_{i}$ so as to decrease some of the numbers $n_{i}$.

It now suffices to suppose that we have a disk $D$ as above. Let $\mu_{1}$ and $\mu_{2}$ be arcs in $D_{i} \cap N(K)$ which join the end-points of $\gamma$ to $K$, with (int $\left.\mu_{1}\right) \cap K=\left(\right.$ int $\left.\mu_{2}\right) \cap K=\varnothing$. Clearly $\mu_{1} \cap K$ and $\mu_{2} \cap K$ lie in two different components of $D_{i} \cap K$. An embedded disk $D^{\prime}$ in $N(K)$ can be found with $\partial D^{\prime}=\mu_{1} \cup \lambda \cup \mu_{2} \cup \lambda^{\prime}$, where $\lambda^{\prime}$ is an arc in $K$ and $K \cap \operatorname{int} D^{\prime}=\varnothing$. To see this we identify $N(K)$ with the mapping cylinder of the double covering of $K$. Then the mapping cylinder restricted to $\lambda$ intersects $K$ in a singular arc, in general. However the singularities may be pushed off the ends of this arc to give $D^{\prime}$.

Let $D_{0}=D \cup D^{\prime}$ and let $\gamma^{\prime}$ denote the arc $\mu_{1} \cup \gamma \cup \mu_{2}$. Then $\partial D_{0}=\gamma^{\prime} \cup \lambda^{\prime}$. We perform an elementary surgery on $K$ along the path $\gamma^{\prime}$. Consequently the effect of this is to add a trivial handle to $K$. Also $\gamma^{\prime}$ is contained in $D_{i}$ and without loss of generality, $D_{i}$ can be chosen so that $\gamma^{\prime}$ joins $\partial D_{i}$ to some curve $\partial D_{j}$ for $j \neq i$. We now observe that a set of compressing disks for $K$ with the trivial handle along $\gamma^{\prime}$ added, can be obtained by replacing $D_{i}$ by two disks $D_{i}^{\prime}, D_{i}^{\prime \prime}$ which have complexities both strictly less than $n_{i}$. For the choices of $D_{i}^{\prime}, D_{i}^{\prime \prime}$, see Figures 2 and 3 .

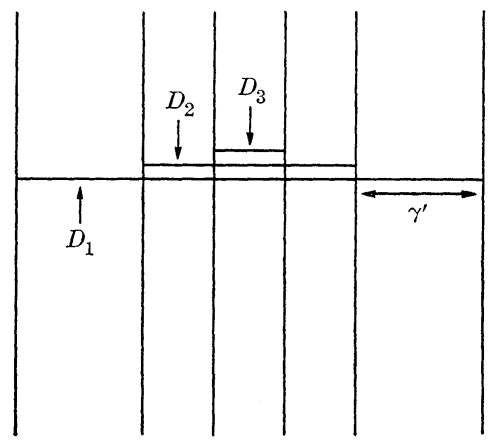

The vertical line are in $K$.

The horizontal lines represent compressing disks $D_{1} \supset D_{2} \supset D_{3}$. 


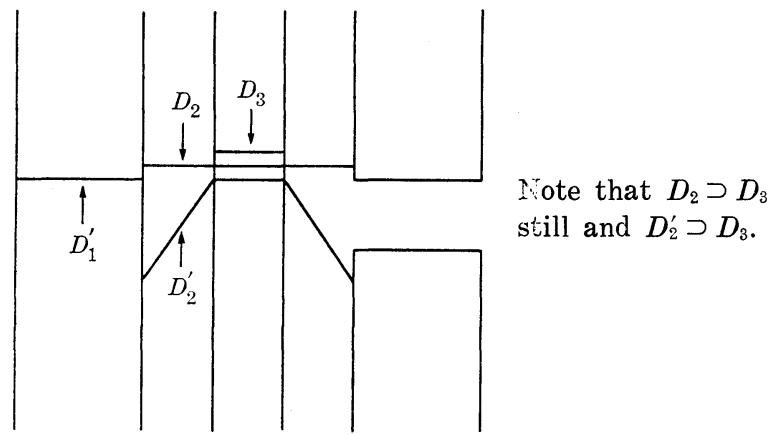

Figure 3

By induction, this completes the proof that all the complexities can be reduced to zero. In this case it can be assumed that $K$ is obtained from $K^{\prime}$ by elementary surgeries along a collection $\Gamma$ of arcs, each of which intersects $K^{\prime}$ precisely at its endpoints.

Now $M$ can be triangulated so that $K$ is a full subcomplex and the 1-skeleton contains $\Gamma$. Applying the procedure of Theorem 1, we add a suitably shifted boundary of a small neighorhood of the 1-skeleton of $M-K$ to $K$, and similarly for $K^{\prime}$ using the 1-skeleton of $M-K-\Gamma$. The same new splitting $\left(M, K^{\prime \prime}\right)$ is obtained in both cases, and $\left(M, K^{\prime \prime}\right)$ is stably equivalent to $(M, K)$ and $\left(M, K^{\prime}\right)$ by [6]. Hence the theorem is proved.

\section{Mappings of degree 1 .}

Lemma 17. Let $f: M^{\prime} \rightarrow M$ be an odd degree map between closed orientable 3-manifolds. If $(M, K)$ is a one-sided Heegaard splitting then there is a one-sided Heegaard splitting $\left(M^{\prime}, K^{\prime}\right)$ and a map $f^{\prime}$ homotopic to $f$ such that $f^{\prime-1}(K)=K^{\prime}$.

Proof. Using elementary surgery, we can obtain $f^{-1}(K)$ is connected by a homotopy of $f$, since $\pi_{1}(M, K)=\{1\}$. Suppose $f^{-1}(K)$ is orientable. Then $f$ factors through the double cover of $M$ associated with the splitting $(M, K)$. This contradicts the assumption that $f$ is of odd degree. Therefore $f^{-1}(K)$ is nonorientable.

We now do the procedure of Theorem 1 using $M^{\prime}$ and the surface $f^{-1}(K)$. As $\pi_{1}(M, K)=\{1\}$, all the 1-handles can be added to $f^{-1}(K)$ by a homotopy of $f$. If $f^{\prime}: M^{\prime} \rightarrow M$ is the new map then $f^{\prime-1}(K)=K^{\prime}$ gives a one-sided Heegaard splitting of $M^{\prime}$ as required.

TheOREM 18. With the hypotheses of Lemma 17, suppose that $f$ is of degree 1 . Then $f^{\prime}$ and $K^{\prime}$ can be chosen so that

$$
f^{\prime}: M^{\prime}-\operatorname{int} N\left(K^{\prime}\right) \longrightarrow M-\operatorname{int} N(K)
$$


is standard (in the sense of [9]), where $f^{\prime-1}(N(K))=N\left(K^{\prime}\right)$.

Proof. The map $f^{\prime}$ found in Lemma 17 lifts to a degree 1 map $\tilde{f}^{\prime}: \widetilde{M}^{\prime} \rightarrow \tilde{M}$, where $\widetilde{M}^{\prime}, \tilde{M}$ are the double covers associated with the splittings $\left(M^{\prime}, K^{\prime}\right),(M, K)$. If $\widetilde{K}, \widetilde{K}^{\prime}$ are the lifts of $K, K^{\prime}$ to $M, M^{\prime}$ respectively then $\widetilde{f}^{\prime-1}(\widetilde{K})=\widetilde{K}^{\prime}$ and $(\widetilde{M}, \widetilde{K}),\left(\widetilde{M}^{\prime}, \widetilde{K}^{\prime}\right)$ are Heegaard splittings.

Let $Y_{1}^{\prime}, Y_{2}^{\prime}$ be the closures of the components of $\tilde{M}^{\prime}-\widetilde{K}^{\prime}$. In Theorem 2.1 of [9] it is proved that $\widetilde{f}^{\prime}$ can be made standard on the handlebodies $Y_{1}^{\prime}, Y_{2}^{\prime}$ by the operation of drilling holes. It is only necessary to do this in a manner which is invariant under the covering transformation to obtain the result.

\section{REFERENCES}

1. G. Bredon and J. Wood, Nonorientable surfaces in orientable 3-manifolds, Invent. Math., 7 (1969), 83-110.

2. W. Heil, Almost sufficiently large Seifert fiber spaces, Michigan Math. J., 20 (1973), 217-223.

3. J. Hempel, 3-Manifolds, Annals of Math. Studies, No. 86, Princeton, 1976.

4. J. H. Rubinstein, On 3-manifolds that have finite fundamental group and contain Klein bottles, to appear in Trans. Amer. Math. Soc.

5. H. Seifert, Topologie dreidimensionaler gefaserter Räume, Acta Math., 60 (1933), 147-238.

6. J. Singer, Three-dimensional manifolds and their Heegaard diagrams, Trans. Amer. Math. Soc., 35 (1933), 88-111.

7. J. Stallings, On the loop theorem, Annals of Math., 72 (1960), 12-19.

8. F. Waldhausen, On irreducible 3-manifolds which are sufficiently large, Annals of Math., 87 (1968), 56-88.

9. - On Mappings of Handlebodies and of Heegaard Splittings, Topology of Manifolds, Markham, Chicago 1970, 205-211.

10. — Heegaard-Zerlegungen der 3-sphäre, Topology 7 (1968), 195-203.

Received January 15, 1977 and in revised form June 23, 1977. The author was supported by a Rothman's Fellowship during this research.

UNIVERSity OF MELbOURNE

Parkville, Victoria, 3052, Australia 


\section{PACIFIC JOURNAL OF MATHEMATICS}

\section{EDITORS}

RICHARD ARENS (Managing Editor)

University of California

Los Angeles, California 90024

C. W. CURTIS

University of Oregon

Eugene, OR 97403

C. C. MOORE

University of California

Berkeley, CA 94720

\section{J. DUGUNDJI}

Department of Mathematics University of Southern California Los Angeles, California 90007

R. Finn aNd J. Milgram Stanford University Stanford, California 94305

\section{ASSOCIATE EDITORS}

E. F. BeCKenbaCH

B. H. NeumanN

F. WOLF

K. YosHIDA

\section{SUPPORTING INSTITUTIONS}

UNIVERSITY OF BRITISH COLUMBIA CALIFORNIA INSTITUTE OF TECHNOLOGY UNIVERSITY OF CALIFORNIA MONTANA STATE UNIVERSITY UNIVERSITY OF NEVADA, RENO NEW MEXICO STATE UNIVERSITY OREGON STATE UNIVERSITY UNIVERSITY OF OREGON
UNIVERSITY OF SOUTHERN CALIFORNIA STANFORD UNIVERSITY UNIVERSITY OF HAWAII UNIVERSITY OF TOKYO UNIVERSITY OF UTAH WASHINGTON STATE UNIVERSITY UNIVERSITY OF WASHINGTON 


\section{Pacific Journal of Mathematics}

\section{Vol. 76, No. $1 \quad$ November, 1978}

Ata Nuri Al-Hussaini, Potential operators and equimeasurability ......... 1

Tim Anderson and Erwin Kleinfeld, Semisimple nil algebras of type $\delta . \ldots .99$

Stephen LaVern Campbell, Linear operators for which $T^{*} T$ and $T+T^{*}$

commute. III ......................................

Robert Jay Daverman, Special approximations to embeddings of codimension one spheres...............................

Donald M. Davis, Connective coverings of $\mathrm{BO}$ and immersions of projective

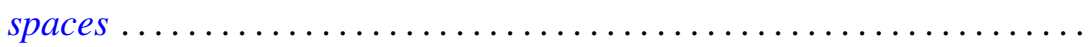

V. L. (Vagn Lundsgaard) Hansen, The homotopy type of the space of maps of

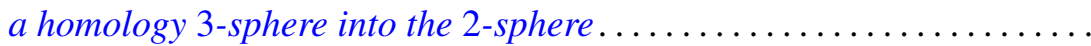

James Victor Herod, A product integral representation for the generalized

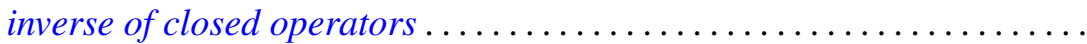

A. A. Iskander, Definability in the lattice of ring varieties ..............

Russell Allan Johnson, Existence of a strong lifting commuting with a

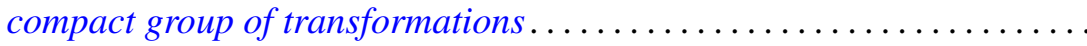

Heikki J. K. Junnila, Neighbornets...................... 83

Klaus Kalb, On the expansion in joint generalized eigenvectors . ......... 109

F. J. Martinelli, Construction of generalized normal numbers . . . . . . . . . 117

Edward O'Neill, On Massey products ....................... 123

Vern Ival Paulsen, Continuous canonical forms for matrices under unitary

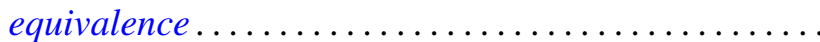

Justin Peters and Terje Sund, Automorphisms of locally compact groups . . . 143

Duane Randall, Tangent frame fields on spin manifolds . . . .

Jeffrey Brian Remmel, Realizing partial orderings by classes of co-simple sets . . . .

J. Hyam Rubinstein, One-sided Heegaard splittings of 3-manifolds ...

Donald Charles Rung, Meier type theorems for general boundary approach

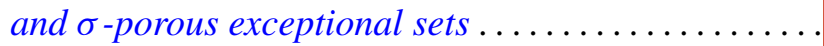

Ryōtarō Satō, Positive operators and the ergodic theorem

Ira H. Shavel, A class of algebraic surfaces of general type constructed from

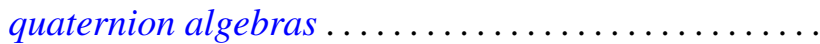

Patrick F. Smith, Decomposing modules into projectives and injectives ....

Sergio Eduardo Zarantonello, The sheaf of outer functions in the polydisc... 